${ }^{1}$ Ondokuz Mayis University, Samsun, Turkey

${ }^{2}$ Amasya University, Amasya, Turkey

\section{Correspondence:}

Pervin Karlı

Address: Amasya University, Amasya, Turkey

Email: parpi2300@hotmail.com

Received: 29.05.2019,

Accepted: 06.08.2019

https://doi.org/10.5799/jcei/5903

\title{
The Importance of Serum Prolidase Activity in Endometriosis
}

\author{
Mukaddes Demir Dural ${ }^{1}$, Fatma Devran Bıldırcın ${ }^{1}$, Pervin Karl ${ }^{2 *}$, Ayse Zehra Özdemir ${ }^{1}$
}

\begin{abstract}
Objective: In this study, our objective was to evaluate the abnormal collagen destruction and turnover and the prolidase activity in the etiopathogenesis of endometriosis, which may deteriorate the collagenous structure of exracellular matrix (ECM).
\end{abstract}

Materials and Methods: For the assessment of the prolidase activity, venous blood samples were obtained from 37 patients, who had applied to the outpatient department of the Medical Faculty at Ondokuz Mayıs University with complaints of pelvic pain, dysmenorrhea and infertility between October 1st, 2011 and February 15th, 2012, underwent clinical and ultrasonographic examination, prediagnosed with endometriosis, and scheduled for laparoscopy or laparotomy. A total of 22 patients, who were diagnosed with endometriosis via intraoperative exploration and/or pathological examination, constituted the study group and the remaining 15 patients, who did not have any pathological finding or were diagnosed with a benign disease except for endometriosis, constituted the control group.

Serum samples obtained from all patients were first centrifuged and then stored in a freezer at $-70 \mathrm{C}$ until the time of analysis. During the analysis, the prolidase activity was measured following the necessary biochemical enzymatic processing.

Results: We found a statistically significant difference between stage 4 and stage 1,2,3 patients in the study group regarding the CA 125 levels $(\mathbf{p = 0 . 0 1 8 )}$. On the other hand, there was no statistically significant difference between stage $1,2,3$ patients in the study group and the control group for serum prolidase levels $(p=0.778)$. There was a statistically significant difference between stage 4 endometriosis patients in the study group and the control group considering the serum prolidase levels $(\mathbf{p}=\mathbf{0 . 0 2 6})$.

Conclusion: We conclude that serum prolidase activity has a critical function in the development of the endometriotic lesions. In endometriosis patients, the increase in the serum prolidase activity may play an important role in the progress to more advanced stages and in the development of infertility.

Keywords: serum prolidase activity, endometriosis, CA 125

\section{INTRODUCTION}

Endometriosis is defined as the presence of endometrial gland and stroma outside the uterus cavity and muscular layer [1]. The prevalence of endometriosis in the general population is unknown [1-3]. The determination of the prevalence is rather difficult because of the asymptomatic patients and nonspecific and diverse symptomatology in symptomatic patients. Approximately $12 \%-32 \%$ of patients, who underwent laparoscopy due to the pelvic pain, $9 \%-50 \%$ of patients, who underwent laparoscopy due to infertility and $50 \%$ of patients, who underwent laparoscopy due to the chronic pelvic pain or dysmenorrhea, were diagnosed with endometriosis [1-3]. The pathological process in endometriosis is not fully elucidated yet. Although the pathogenesis is not known, several theories based on certain evidence were proposed [1]. Retrograde menstruation - transplantation, coelomic metaplasia - induction, vascular dissemination and theories related to the immune system are among them. As endometriosis is usually encountered in women of reproductive age, the symptoms 
regress with the onset of menopause [4]. Reoccurrence of symptoms in postmenopausal women treated with estrogen replacement therapy indicates that endometriosis is an estrogen-dependent disease. The immune system hypothesis explains the secretion of different cytokines, which initiate the proliferation of implants, lead to the capillary support and to leukocyte chemotaxis due to the peritoneal inflammation following the access of the endometrial implant and inflammatory cells to the peritoneal cavity [4]. Oxidative stress might be one of the components of the inflammatory process [5]. Therefore, the immune system may play an important role in the characteristics and the severity of the clinical findings and in whom they will emerge $[6,7]$. For a long time, it is widely accepted that there is a familial disposition in endometriosis. Pain is the most common symptom of endometriosis. Approximately $75 \%$ of the symptomatic patients complain of pelvic pain and/or dysmenorrhea [8]. Chronic pelvic pain, dysmenorrhea, dyspareunia, subfertility, abnormal menstrual bleeding, and back pain are other symptoms. The stage of endometriosis does not correlate with the presence and the severity of the symptoms. Laparoscopy and visual detection of the lesion are the gold standards in the diagnosis of endometriosis [810]. Endometriosis is a chronic disease, which requires lifelong optimal treatment and avoidance from the reoperation. In spite of intensive research, the optimal treatment of endometriosis is still not clear. The treatment of endometriosis should be based on the specific symptoms, the severity of the symptoms, the localization of the endometriotic lesions, the goals of the treatment, the age of the patient and the desire to preserve the fertility in the future. Observation and the medical treatments consisting of hormonal suppression and analgesia and surgical interventions are the main therapeutic alternatives.

Under normal physiological conditions, the endometrium tissue is formed by the extracellular matrix (ECM). The matrix metalloproteinase (MMP) consists of proteolytic enzymes, which regulate and change ECM. Studies demonstrated that endometriosis may develop as a result of the impairment of the regulatory control in MMP $[11,12]$. Regarding the collagen remodeling in ECM, which is started by MMPs, collagen degradation stimulated by prolidase is the final step. The abnormal collagen degradation and turnover and increased prolidase activity, which may deteriorate the collagenous structure of ECM, may be responsible for the etiopathogenesis of endometriosis [13].

It was demonstrated that MMP-9 increased in the ectopic endometrium of women with endometriosis [14]. The MMP activity is controlled by oxidative stress activity. It was reported that the reactive oxygen species had an important role in the invasion and growth of the endometrial cells in the peritoneal cavity in women with endometriosis $[15,16]$. It was also determined that MMPs had a critical function in the early development of the endometriotic lesions and
MMP inhibitors prevented the endometriotic lesions $[17,18]$.

The activity of matrix metalloproteinases and prolidases was investigated in various obstetric and gynecological diseases. It was observed that MMP-9, MMP-2, MMP-3, and collagenases increased in the fetal membrane and amniotic fluid close to the delivery. It was suggested that the MMP activation in the specific areas of the fetal membrane may cause membrane rupture at term or preterm [19-22]. MMPs participate in the placenta invasion. The increase in MMP levels is one of the factors blamed for the placental abruption $[23,24]$. The increase in the extracellular matrix turnover is one of the pathological mechanisms responsible for the vascular resistance and emergence of the endothelial dysfunction in the intrauterine growth retardation. Therefore the serum prolidase level, which is a marker for the extracellular matrix and collagen turnover, was investigated in the intrauterine growth retardation and it was demonstrated that it increased in these cases [25].

The objective of our study was to evaluate the abnormal collagen degradation and turnover in the etiopathogenesis of endometriosis and the prolidase activity, which may deteriorate the collagenous structure in ECM. Furthermore, the elucidation of the etiology may facilitate the development of new therapeutic approaches in endometriosis.

\section{MATERIALS AND METHODS}

This article was approved by the Ethics Committee of the Faculty of Medicine of Ondokuz Mayis University (OMU TAEK 2010-153). Study type is "prospective study". For the assessment of the prolidase activity, venous blood samples were obtained from 37 patients, who had applied to the outpatient department of the Medical Faculty at Ondokuz Mayis University with complaints of pelvic pain, dysmenorrhea and infertility between October 1st, 2011 and February 15th, 2012, underwent clinical and ultrasonographic examination, prediagnosed with endometriosis, and scheduled for laparoscopy or laparotomy. Written patient consent was obtained from all patients in the study and control groups. A total of 22 patients, who were diagnosed with endometriosis via intraoperative exploration and/or pathological examination, constituted the study group and the remaining 15 patients, who did not have any pathological finding or were diagnosed with a benign disease except for endometriosis, constituted the control group. All patients in the study and control groups were Caucasians and were Turkish nationality. The educational background was not taken into consideration. All patients in the study and control group fulfilled the patient information form and the following patient characteristics were recorded: Age, presence and duration infertility, pre-operative CA 125 values, presence of dysmenorrhea and dyspareunia symptoms, previous endometriosis operations, presence of concomitant gynecological diseases, stage of the disease, operation 
reports, primary complaints, information about preoperative TVS, gravida, parity, number of abortion and living children, drug therapy, presence of chronic diseases was recorded. Patients with endometriosis, who were diagnosed with laparoscopy or laparotomy and included in the study, were classified according to the endometriosis classification of ASRM (American Society for Reproductive Medicine): Stage 1 (minimal), stage 2 (mild), stage 3 (moderate), and stage 4 (severe). In the study group, 2 patients were at stage 1 (minimal), one patient at stage 2 (mild), 4 patients at stage 3 (moderate), and 15 patients at stage 4 (severe). Five and 17 of the patients underwent laparotomy and laparoscopy respectively.

\section{Inclusion Criteria}

a) 18-47 years of age

b) preoperative examination and suspected unilateral or bilateral ovarian endometrioma by ultrasound and diagnosed as endometrioma in the pathology after laparoscopic surgery

\section{Exclusion Criteria}

a) ovarian surgery, hysterectomy or determined asprevious surgery

b) postoperative non-endometrioma pathology

c) hormonal preoperative get treatment or have any hormonal disease

d) inflammatory bowel disease or irritable colon syndrome

\section{Obtainment of Serum Samples}

Serum samples were taken from the left or right antecubital veins of patients while they were sitting at room temperature $\left(24^{\circ} \mathrm{C}\right)$ after the related region was cleaned with alcohol and cotton. A 10cc syringe was used and approx. 7-8 cc blood was drawn. The samples were taken approx. one hour before the intervention. The blood samples were transferred in vacuumed and red-capped biochemistry tubes, which did not contain an anticoagulant agent. After the centrifuge process, samples were stored at $-70^{\circ} \mathrm{C}$ until the analysis.

\section{Serum Prolidase Enzyme Activity}

The prolidase enzyme activity was calculated with the measurement of the L-proline complex, which forms as a result of the degradation of the glycine-L-proline substrate with enzymes, and ninhydrin at $90^{\circ} \mathrm{C}(\mathrm{pH}: 7.8)$. The analysis was performed according to the modified Chinard method with the reagents produced in the Research Laboratory of the Biochemistry Department in the Ondokuz Mayıs University Medical Faculty [26]. The serum prolidase enzyme activity was assessed by a specialist, who was blinded to the study and control groups.

\section{The Preparation of the Solutions}

1. $50 \mathrm{mM}$ Tris- $\mathrm{HCl}$ tamponade ( $\mathrm{pH}: 7.8)$ : After 6.057 $\mathrm{g}$ Tris was completely solved in approx. $60 \mathrm{ml}$ distilled water in the magnetic mixer, $\mathrm{pH}$ was adjusted to 7.8 with $1 \mathrm{~N} \mathrm{HCl}$ and the volume was adjusted to 1 liter with distilled water.

2. $5 \mathrm{mM} \mathrm{MgCl} 2-4 \mathrm{H} 2 \mathrm{O}, 0.1 \%$ Triton X-100, $1 \mathrm{mM}$ GSH: In a dark-colored bottle containing approx. $60 \mathrm{~mL} 50$ $\mathrm{mM}$ Tris- $\mathrm{HCl}$ tamponade ( $\mathrm{pH}: 7.8), 100 \mathrm{mg} \mathrm{MnCl} 2-4 \mathrm{H} 2 \mathrm{O}$, $100 \mu \mathrm{l}$ Triton X-100 and $30.7 \mathrm{mg}$ GSH were dissolved in this order in a magnetic mixer. The final volume was adjusted to $100 \mathrm{~mL}$ with $50 \mathrm{mM}$ Tris- $\mathrm{HCl}$ tamponade (pH: 7.8).

3. $144 \mathrm{mM}$ glycine-L-proline (substrate): First $5 \mathrm{mg}$ $\mathrm{MnCl} 2-4 \mathrm{H} 2 \mathrm{O}$ and then $124 \mathrm{mg}$ glycine-L-proline were dissolved completely in a dark-colored bottle containing approx. $3 \mathrm{ml} 50 \mathrm{mM}$ Tris- $\mathrm{HCl}$ tamponade (pH: 7.8). Final volume was adjusted to $5 \mathrm{ml}$ with $50 \mathrm{mM}$ Tris- $\mathrm{HCl}$ buffer (pH: 7.8).

4. $\quad 0.45 \mathrm{M}$ TCA: After $73.52 \mathrm{~g}$ trichloroacetic acid (TCA) was completely dissolved in $600 \mathrm{ml}$ distilled water in the magnetic mixer, the final volume was adjusted to $1000 \mathrm{ml}$ with distilled water.

5. L-proline standard: After $7.5 \mathrm{mg}$ L-proline was completely dissolved in approx. $60 \mathrm{ml} 0.45 \mathrm{M}$ TCA in a magnetic mixer, the final volume was adjusted to $100 \mathrm{ml}$ with distilled water.

6. Modified Chinard reagent: $550 \mathrm{ml}$ glacial acetic acid (GAA) and $450 \mathrm{ml} 6 \mathrm{M}$ orthophosphoric acid (OFA) were mixed in a vortex mixer until the mixture became completely homogeneous. $60 \mathrm{ml}$ of this mixture was transferred into a dark-colored bottle and $30 \mathrm{~g}$ ninhydrin was added. After it was completely dissolved in the magnetic mixer at $70^{\circ} \mathrm{C}$, the final volume was adjusted to $100 \mathrm{ml}$ with the GAA-OFA mixture.

\section{Implementation of the Method}

Serum samples, which were kept in the deep freezer, were brought to room temperature. Then they were diluted with Tris- $\mathrm{HCl}$ tamponade containing $5 \mathrm{mM} \mathrm{MnCl} 2-4 \mathrm{H} 2 \mathrm{O}, 0.1 \%$ Triton X-100 and $1 \mathrm{mM}$ GSH in a ratio of 1:6 and stored at $37^{\circ} \mathrm{C}$ to incubate for 3 hours. Then, $100 \mu$ from each sample were transferred to tubes, which were labeled as sample and blind sample. $100 \mu \mathrm{l}$ substrate (glycine-L-proline) was added to the sample tube and the sample and blind sample tubes let to incubate at $37^{\circ} \mathrm{C}$ for 30 minutes. After the incubation, 1 $\mathrm{ml} 0.45 \mathrm{M}$ TCA was added to both tubes and the prolidase enzyme was inhibited with mixing in the vortex mixer. Then, $100 \mu \mathrm{l}$ substrate was added and mixed to the blind sample tube, the sample was centrifuged at $4000 \mathrm{rpm}$ for 10 minutes. After the centrifuge, $0.5 \mathrm{ml}$ supernatant from sample and blind sample was transferred to new tubes. $0.5 \mathrm{ml}$ standard (7.5\% mg L-proline) and $0.5 \mathrm{ml}$ TCA were added to separate tubes and labeled as standard and reagent blind respectively. $1 \mathrm{~mL}$ GAA added to all tubes and mixed in the vortex mixer and then $1 \mathrm{ml}$ modified Chinard reagent added to the tubes and mixed again. All tubes let to incubate at $90^{\circ} \mathrm{C}$ for 20 minutes. After the incubation period, all tubes brought to room temperature and the absorbance at $515 \mathrm{~nm}$ wavelength 
Serum Prolidase Activity and Endometriosis

\begin{tabular}{|l|}
\hline \multicolumn{1}{|c|}{$\frac{\mathbf{A}_{\mathbf{o}} \mathrm{S}-\mathbf{A}_{\mathbf{o}} \mathrm{BS}-\mathbf{A}_{\mathbf{o}} \mathrm{BR}}{\mathbf{A}_{\mathbf{o}} \mathrm{S}-\mathbf{A}_{\mathbf{o}} \mathrm{BR}} \times 1560$} \\
\hline $\mathbf{A}_{\mathbf{o}} \mathrm{S}=$ Absorbance of Sample \\
\hline $\mathbf{A}_{\mathbf{o}} \mathrm{BS}=$ Absorbance of Blind Sample \\
$\mathbf{A}_{\mathbf{o}} \mathrm{BR}=$ Absorbance of Blind Reagent \\
\hline $\mathbf{A}_{\mathbf{o}} \mathrm{S}=$ Absorbance of Standard \\
\hline
\end{tabular}

Figure 1. Calculation of Serum Prolidase Enzyme Activity

Table 1. Distribution of the cases according to the stage of endometriosis

\begin{tabular}{ccc}
\hline \multicolumn{4}{c}{ Stage Number of patients \% } \\
\hline 1 & 2 & 9.3 \\
\hline 2 & 1 & 4.5 \\
\hline 3 & 4 & 18.1 \\
\hline 4 & 15 & 68.1 \\
\hline
\end{tabular}

was measured with the spectrophotometer. The obtained data were inserted to the below-mentioned formula and the serum prolidase enzyme activities were calculated in $\mathrm{U} / \mathrm{l}$ (Figure 1).

\section{Statistical Analysis}

All analyses were done with SPSS for Windows v15.0. The intergroup comparisons were performed with the Student's t-test, Mann-Whitney U test, and Chi-square test.

\section{RESULTS}

The preoperative CA 125 values were between 5 and 150 $\mathrm{IU} / \mathrm{ml}$ in the study group. The duration of infertility was between 2 and 11 years among patients, who were diagnosed with infertility. Eighteen of the patients had symptoms related to dysmenorrhea and 10 patients had symptoms related to dyspareunia. Eight patients had previous endometriosis operation. Five patients had myoma uteri as a concomitant gynecological pelvic disease. 10 of the patients, who were diagnosed with endometriosis via laparoscopy and laparotomy, were operated due to the pre-diagnosis of pelvic pain, a pelvic mass. Twelve patients were operated due to the prediagnosis of infertility (8 patients had primary infertility, 4 patients had secondary infertility).

Total abdominal hysterectomy and bilateral salpingooophorectomy were performed in one of 15 stage 4 patients. total abdominal hysterectomy and unilateral salpingooophorectomy were performed one of these patients. Eight patients underwent endometrioma cyst excision and adhesiolysis. Five patients underwent endometrioma cyst aspiration, cyst capsule ablation, and adhesiolysis.

Four stage 3 patients underwent adhesiolysis and endometrioma cyst excision. We did not perform any additional intervention during laparoscopy in patients with stage 1 or stage 2 . Drug treatment with a $\mathrm{GnRH}$ agonist was initiated in one of stage 4 patients in the postoperative period.
Table 2. Comparison in the study group according to the symptom and fertility characteristics

\begin{tabular}{lccc}
\hline Symptom & \multicolumn{2}{c}{$\begin{array}{c}\text { Number of } \\
\text { patients (\%) }\end{array}$} & P \\
\hline Infertility (No) & 6 & $(17.2)$ & \multirow{2}{*}{0.033} \\
\hline Infertility (Yes) & 16 & $(72.8)$ & \\
\hline Dysmenorrhea (No) & 5 & $(22.7)$ & \multirow{2}{*}{0.011} \\
\hline Dysmenorrhea (Yes) & 17 & $(77.3)$ & \\
\hline Dyspareunia (No) & 12 & $(54.5)$ & \multirow{2}{*}{0.670} \\
\hline Dyspareunia (Yes) & 10 & $(45.5)$ & \\
\hline Infertility & 14 & $(63.6)$ & \multirow{2}{*}{0.201} \\
\hline Abdominal pain & 8 & $(36.4)$ & \\
\hline
\end{tabular}

There was no statistically significant difference between the study and control groups regarding the age of the participants. The age of the patients was between 18 and 47 years in the study group and between 18 and 45 years in the control group. The mean age was $31 \pm 9.1$ and $28 \pm 2.4$ years in the study and control groups respectively.

Sixteen patients in the study group had infertility and 6 had no infertility. The comparison of the patients in the study group for infertility revealed a statistically significant difference $(\mathrm{p}<0.05)$. In the study group, 17 patients had dysmenorrhea and 5 patients had no dysmenorrhea. The comparison of the patients in the study group for dysmenorrhea showed a statistically significant difference $(\mathrm{p}=0.011)$. Again in the study group, 10 patients had dyspareunia and 12 had no dyspareunia. The comparison of the patients in the study group for dyspareunia showed no statistically significant difference $(p>0.05)$. In the study group, the main symptom was infertility in 14 patients and abdominal pain in 8 patients. The comparison of the patients in the study group for abdominal pain and infertility displayed no statistically significant difference $(\mathrm{p}<0.05)$ (Table 2). In the study group, pelvic masses (size: $2-9 \mathrm{~cm}$ ) were determined in 19 patients during TVS. Eight of these pelvic masses were unilateral and 11 were bilateral. Twentytwo patients underwent laparoscopy/laparotomy and were diagnosed with endometriosis according to the ASRM (American Society for Reproductive Medicine) endometriosis classification and were included in the study group. Ten of the endometriosis patients (45.4\%), who were prediagnosed during laparoscopy/laparotomy, were operated due to the chronic pelvic pain and pelvic mass, 12 of them (54.6\%) were operated due to the prediagnosis of infertility ( 8 patients had primary infertility and 4 had secondary infertility). 15 of these 22 patients $(68.1 \%)$ had stage 4 endometriosis. Two of the cases $(9.3 \%)$ had stage 1 (minimal), one (4.5\%) had stage 2 (mild), and four (18.1\%) had stage 3 (moderate) endometriosis (Table 1 ).

Stage 4 and stage 1, 2, 3 patients in the study groups were compared for serum CA 125 levels with a statistically significant difference $(\mathrm{p}=\mathbf{0 . 0 1 8})$ (Table 3$)$. 
Serum Prolidase Activity and Endometriosis

Table 3. Comparison of stage 4 and stage 1,2,3 endometriosis patients for serum CA 125 level

\begin{tabular}{llcccc}
\hline & Stage & Patient & Mean & Min./Max. & P \\
\hline \multirow{3}{*}{ Preoperative CA 125} & Stage $(1,2,3)$ & 7 & $29.4 \pm 21.5$ & $5-66$ & 0.018 \\
\cline { 2 - 6 } & Stage 4 & 15 & $61.7 \pm 32.3$ & $5-50$ & $5-150$ \\
\cline { 2 - 6 } & Total & 22 & $51,432.6$ & & \\
\hline
\end{tabular}

Table 4. Comparison of stage 1,2,3 and stage 4 endometriosis patients in the study group for the serum prolidase levels

\begin{tabular}{|c|c|c|c|c|}
\hline Group & $\begin{array}{c}\text { Number of } \\
\text { patients }\end{array}$ & $\begin{array}{l}\text { Mean } \pm S D \\
\text { Prolidase }\end{array}$ & Median & $\begin{array}{c}\text { Max./Min. } \\
\text { Prolidase }\end{array}$ \\
\hline Stage 4 endometriosis & 15 & $915 \pm 297$ & 938 & $1428-457$ \\
\hline Stage $1,2,3$ endometriosis & 7 & $720 \pm 268$ & 672 & $1079-287$ \\
\hline Control Group & 15 & $697 \pm 203$ & 669 & $1023-208$ \\
\hline & & & & $\underline{\mathbf{P}}$ \\
\hline & \multicolumn{3}{|c|}{ Between stage 4 and the control group } & 0.026 \\
\hline & \multicolumn{3}{|c|}{ Between stage $1,2,3$ and the control group } & 0.778 \\
\hline & \multicolumn{3}{|c|}{ Between stage 1,2,3 and stage 4} & 0.259 \\
\hline
\end{tabular}

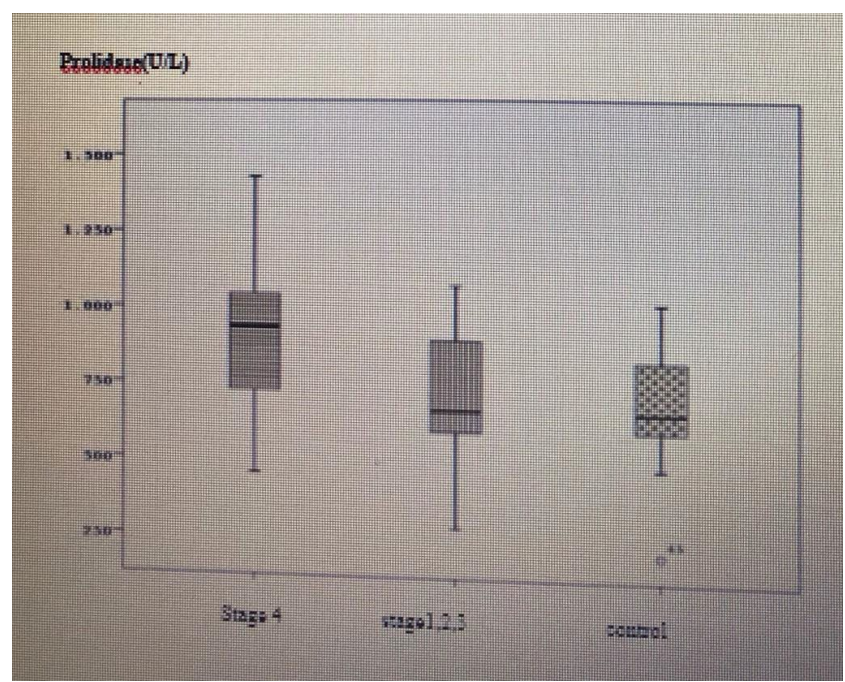

Figure 2. The distribution of the serum prolidase levels between the stage 1,2,3 and stage 4 endometriosis patients in the study group and the control group

The comparison of stage $1,2,3$ patients in the study with the control group for serum prolidase levels did not display any statistically significant difference $(\mathrm{p}=0.778)$. There was also no statistically significant difference between stage 4 and stage 1,2,3 patients regarding the serum prolidase levels $(\mathrm{p}=0.259)$. However, we found a statistically significant difference between stage 4 endometriosis patients in the study group and the control group in respect of the serum prolidase levels ( $\mathbf{p}=\mathbf{0 . 0 2 6}$ ) (Table 4).

Although the serum prolidase levels were higher in the study group compared to the control group, the difference was not statistically significant $(\mathrm{p}=0.086)$. However, there was a statistically significant difference between stage 4 endometriosis patients in the study group and the control group for the serum prolidase levels $(\mathrm{p}=0.026)$. We could not detect any statistically significant difference between stage 4 and stage 1,2,3 endometriosis patients in the study group regarding the serum prolidase levels (Figure 2).

The comparison of the study and control groups for the serum prolidase levels showed that the stage 4 patients were responsible for the high prolidase levels and the difference was not statistically significant because of the low prolidase levels in stage 1,2,3 patients. In the study group, the comparison of the patients with and without infertility displayed a statistically significant difference for the serum prolidase level $(\mathrm{p}=0.021)$. However, there was no statistically significant difference between the patients with and without abdominal pain for the serum prolidase level $(\mathrm{p}=0.183)$. Similarly, there was also no statistically significant difference between the patients with and without dyspareunia and dysmenorrhea symptoms for the serum prolidase level ( $\mathrm{p}=0.821$ and $\mathrm{p}=0.594$ respectively) (Table 5).

In the study group, 16 patients had infertility and 6 patients did not have infertility. The comparison of the patients with and without infertility for serum prolidase level showed a significant difference $(\mathrm{p}=0.012)$ (Figure 3).

\section{DISCUSSION}

Matrix metalloproteinase (MMP) family is an important member of the extracellular proteinases $[17,18]$. The most important function of MMPs is the degradation of the extracellular matrix (ECM). Studies showed that MMPs took part in various physiological and pathological processes $[17,18]$. These enzymes are rather essential regarding ECM turnover, tissue remodeling, angiogenesis, morphogenesis, and development. MMPs also play an important role in several physiological processes such as embryonic development, ovulation, bone remodeling and wound healing $[17,18,27]$. Furthermore, it is well known that MMPs take part in cell migration, invasion, proliferation, and 
Table 5. Comparison of patients in the study group classified according to the symptoms and fertility characteristics for the serum prolidase levels

\begin{tabular}{|c|c|c|c|c|c|}
\hline Group & $\begin{array}{c}\text { Number of } \\
\text { patients }\end{array}$ & $\begin{array}{l}\text { Mean } \pm S D \\
\text { Prolidase }\end{array}$ & $\begin{array}{l}\text { Median } \\
\text { Prolidase }\end{array}$ & $\begin{array}{c}\text { Min./Max. } \\
\text { Prolidase }\end{array}$ & P (significance) \\
\hline Infertility - No & 6 & $648 \pm 128$ & 613 & $528-815$ & \multirow{2}{*}{0.021} \\
\hline Infertility (Yes) & 16 & $930 \pm 308$ & 969 & $287-1428$ & \\
\hline Abdominal pain - No & 14 & $902 \pm 312$ & 961 & $287-1428$ & \multirow{2}{*}{0.183} \\
\hline Abdominal pain - Yes & 8 & $768 \pm 264$ & 727 & $528-1305$ & \\
\hline Dyspareunia - No & 12 & $811 \pm 234$ & 844 & $287-1079$ & \multirow{2}{*}{.821} \\
\hline Dyspareunia-Yes & 10 & $904 \pm 365$ & 868 & $457-1428$ & \\
\hline Dysmenorrhea - No & 5 & $781 \pm 439$ & 651 & $287-1428$ & \multirow{2}{*}{0.594} \\
\hline Dysmenorrhea-Yes & 17 & $874 \pm 255$ & 874 & $457-1355$ & \\
\hline
\end{tabular}

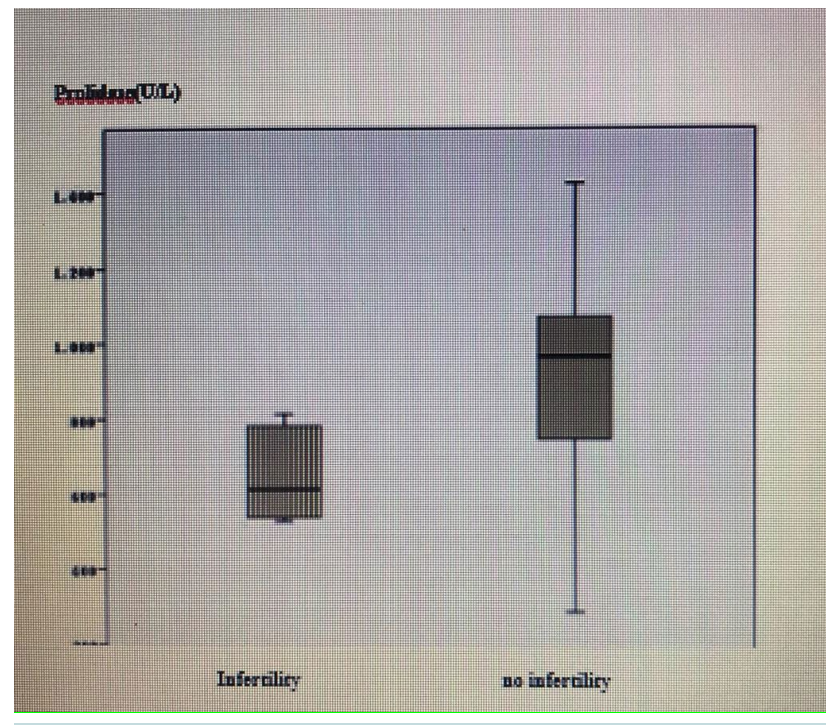

Figure 3. The distribution of serum prolidase levels between the patients with and without infertility in the study group

apoptosis. It is believed that the uncontrolled increases in the MMP activity take part in the pathogenesis of acute and chronic diseases depending on the ECM degradation [27].

The proteolytic activity of MMPs may be prevented with tissue metalloproteinase inhibitors (TIMP), which inhibit the degradation of collagen and other matrix macromolecules. The existing proportion between MMPs and TIMPs may change during various physiological and pathological processes and thus the balance between them may play an important role in the pathogenesis of several pathological events. Numerous processes including ovulation, implantation, gestation, lactation, and involution, which are components of the reproduction process, require TIMP and/or MMP activation [27].

In a study, it was determined that serum prolidase activity was increased and placental prolidase activity was decreased depending on the possible placental use in patients with early pregnancy loss. In this study, the authors concluded that the decreased placental activity of prolidase might be an etiopathological factor in women with early pregnancy loss [28]. In a study conducted by Camuzcuoğlu et al., it was found out that serum prolidase activity and total oxidative stress increased and total antioxidant capacity decreased in women with epithelial ovarian cancer. They also determined that there was a strong correlation between the serum prolidase activity and stage and the grade of the tumor and the preoperative CA 125 levels. This study indicated that serum prolidase activity might be a prognostic factor in epithelial ovarian cancer [29].

Endometriosis was detected in $80 \%$ of women with complaints of pelvic pain or infertility [1-3]. Factors like the immune, hormonal, genetic, and environmental factors of the peritoneal fluid and content and invasive potential of the endometriotic cells are determinative in the development of endometriosis [30]. Oxidative stress might be one of the components of the inflammatory process [5].

Regarding the etiology of endometriosis, which is not clear yet, various agents were blamed. One of the most popular opinions is that the matrix metalloproteinases (MMP) play an important role in the etiology of endometriosis [11,12]. During the normal physiological processes, endometrium tissue is formed by the extracellular matrix (ECM). MMPs are proteolytic enzymes that regulate and modify ECM $[11,12]$. Studies had shown that the impairment in the regulatory control of MMPs might lead to endometriosis $[11,12]$. Abnormal collagen degradation and turnover and increased prolidase activity, which may deteriorate the collagenous structure of ECM may be responsible for the etiopathogenesis of endometriosis [13]. In our study, significant outcomes of serum prolidase activity in stage 4 endometriosis may be related to abnormal collagen degradation and turnover and increased prolidase activity.

Oxidative stress is one of the factors blamed in the etiology of endometriosis $[15,16]$. Macrophages, iron ions and the presence of the environmental waste, which cause oxidative stress and endometriosis due to the disturbance of the balance between the radical oxygen species and antioxidants in the peritoneal fluid of some women, are considered $[15,16]$. In several studies, it was demonstrated 
that the reactive oxygen species had an important role in the adhesion and growth of the endometrial cells in the peritoneal cavity of women with endometriosis $[15,16]$.

MMP-9 levels, which is a specific enzyme found in the ectopic endometrium of the women with endometriosis, was increased according to the results of some studies [14]. MMPs may be controlled with oxidative stress. Radical oxygen species (ROS) are responsible for the conversion of MMP-9 and MMP-2 from latent form to the active form. Animal studies had shown that the use of the antioxidant enzyme might be a protective factor against the development of endometriosis. Melatonin has antioxidant activity. In an animal study, it was demonstrated that melatonin had a protective role in the prevention of the peritoneal endometriosis. It was observed that melatonin suppressed the activation and release of MMPs after MMP activity was increased by oxidative stress. Further studies are needed for the demonstration of the role of melatonin in the regression of the endometriotic lesions through the MMP regulation $[15,16]$. Nap et al. conducted an animal study and found out that MMPs had a critical function in the early development of the endometriotic lesions and MMP inhibitors prevented the endometriosis lesions $[17,18]$. Further studies are needed for the definition of the role of the release and regulation of MMPs in the development of endometriosis.

Vural $\mathrm{M}$ et al. claimed that serum prolidase activity is higher in women with fibriods and Hilali et al. found that prolidase activity is different in women with PCOS. It was proven that prolidase enzyme activity may play a role in preeclampsia in 2017 [31,32].

In our study, prolidase level was higher in women with endometriosis compared to the control group. However, the difference was not statistically significant. The comparison of stage 4 endometriosis patients with the control group showed a statistically significant difference for the serum prolidase level. In addition, there was also a statistically significant difference between the women with and without infertility for the serum prolidase level.

\section{CONCLUSION}

In light of the data obtained in our study, we conclude that serum prolidase activity has a critical function in the development of the endometriotic lesions. The elevation of the serum prolidase activity may play an important role in the progress to more advanced stages and in the development of infertility in patients with endometriosis. These results confirmed that increased prolidase activity, which might cause abnormal collagen degradation and turnover and disturbance in the collagenous structure of ECM, might be responsible for the etiopathogenesis of endometriosis.

Declaration of interest: The authors report no conflicts of interest.

Financial Disclosure: No financial support was received.

\section{REFERENCES}

1. Sangi-Haghpeykar H, Poindexter AN 3rd. Epidemiology of endometriosis among parous women. Obstet Gynecol 1995;85:983.

2. Chatman DL, Ward AB. Endometriosis in adolescents. J Reprod Med 1982;27:156.

3. Missmer SA, Hankinson SE, Spiegelman D, et al. Incidence of laparoscopically confirmed endometriosis by demographic, anthropometric, and lifestyle factors. Am J Epidemiol 2004;160:784.

4. Lebovic DI, Mueller MD, Taylor RN. Immunobiology of endometriosis. Fertil Steril 2001;75:1.

5. Van Langendonckt A, Casanas-Roux F, Donnez J. Oxidative stress and peritoneal endometriosis. Fertil Steril 2002;77:861.

6. Harada T, Iwabe T, Terakawa N. Role of cytokines in endometriosis. Fertil Steril 2001; 76:1.

7. Eyster KM, Klinkova O, Kennedy V, Hansen KA. Whole genome deoxyribonucleic acid microarray analysis of gene expression in ectopic versus eutopic endometrium. Fertil Steril 2007;88:1505.

8. Sinaii N, Plumb K, Cotton L, et al. Differences in characteristics among 1,000 women with endometriosis based on extent of disease. Fertil Steril 2008;89:538.

9. Kennedy S, Bergqvist A, Chapron C, et al. ESHRE guideline for the diagnosis and treatment of endometriosis. Hum Reprod 2005;20:2698.

10. Engemise S, Gordon C, Konje JC. Endometriosis. BMJ 2010;340:c2168.

11. Hulboy DL, Rudolph LA, Matrisian LM. Matrix metalloproteinases as mediators of reproductive function. Mol Hum Reprod 1997;3:27-45.

12. Sillem M, Prifti S, Neher M, Runnebaum B. Extra cellular matrix remodelling in the endometrium and its possible relevance to the pathogenesis of endometriosis. Hum Reprod Update 1998;4:730-5.

13. Nap AW, Dunselman GA, de Goeij AF, Evers JL, Groothuis PG. Inhibiting MMP activity prevents the development of endometriosis in the chicken chorioallantoic membranemodel. Hum Reprod 2004;19:2180-7.

14. Collette T, Maheux R, Mailloux J, Akoum A. Increased expression of matrix metalloproteinase- 9 in the eutopicendometrial tissue of women with endometriosis. Hum Reprod 2006;21:3059-67.

15. Arumugam K, Yip YC. De novo formation of adhesions inendometriosis: the role of iron and free radical reactions. Fertil Steril 1995;64:62-4.

16. Alpay Z, Saed GM, Diamond MP. Female infertility and freeradicals: potential role in adhesions and endometriosis. J Soc Gynecol Investig 2006;13:390-8.

17. Woessner JF Jr. Matrix metalloproteinases and theirinhibitors in connective tissue remodeling. FASEB J 1991;5:2145-54. 
18. Murphy G. Matrix metalloproteinases and their inhibitors. Acta Orthop Scand Suppl 1995;266:55-60.

19. Vadillo-Ortega F, Gonzalez-Avilla G, Furth EE, et al. 92 $\mathrm{kd}$ type IV collagenase (matrix metalloproteinase-9) activity in human amniochorion increases with labor Am J Pathol expressed within the placenta during multiple stages of human pregnancy. Plasenta 1996;17:557.

20. Tsatas D, Baker MS, Rice GE. Differantial expression of proteases in human gestational tissues before, during and after spontaneous-onset labour at term. J Reprod Fertil 1999;116:43.

21. Chapron C, Fauconnier A, Dubuisson JB, et al. Deep infiltrating endometriosis: relation between severity of dysmenorrhoea and extent of disease. Hum Reprod 2003; 18:760.

22. D'Hooghe TM, Debrock S, Hill JA, Meuleman C. Endometriosis and subfertility: is the relationship resolved? Semin Reprod Med 2003;21:243.

23. Mackenzie AP, Schatz F, Krikun G, et al. Mechanisms of abruption-induced premature rupture of the fetal membranes: Thrombin enhanced decidual matrix metalloproteinase-3 (stromeylsin-1) expression. Am J Obstet Gynecol 2004;191:1996.

24. Krikun G, Huang ST, Schatz F, et al. Thrombin activation of endometrial endothelial cells: a possible role in intrauterine growth restriction. Thromb Haemost 2007;97:245.
25. Burton GJ, Jauniaux E. Oxidative stres. Best Pract Res Clin Obstet Gynaecol 2011;25:287.

26. Chinard P. Photometric determination of proline and ornithine. J Biol Chem. 1952;199:61-65.

27. Onat T, Emerk K, Sözmen EY. Human Biochemistry, Palme publishing, Ankara, 2002.

28. Toy H, Camuzcuoglu H, Camuzcuoglu A, Celik H, Aksoy N. Decreased serum prolidase activity and increased oxidative stress in early pregnancy loss. Gynecol Obstet Invest 2010;69(2):122-12.

29. Camuzcuoglu H, Arioz DT, Toy H, Kurt S, Celik H, Erel O. Serum paraoxonase and arylesterase activities in patients with epithelial ovarian cancer. Gynecol Oncol 2009;112:481-5.

30. Koninckx PR, Kennedy SH, et al. Endometriotic disease: the role of peritoneal fluid. Hum Reprod Update 1998;4(5):741-51.

31. Hilali N, Vural M, Camuzcuoglu H, Camuzcuoglu A, Aksoy N. Increased prolidase activity and oxidative stress in PCOS. Clinical endocrinology 2013;79(1):105110.

32. Vural M, Camuzcuoglu H, Toy H, Camuzcuoglu A, Aksoy N. Oxidative stress and prolidase activity in women with uterine fibroids. Journal of Obstetrics and Gynaecology 2012;32(1): 68-72. 